\title{
Evaluation of a new left atrial retractor for minimally invasive mitral valve surgery
}

\author{
Olivier Jegaden, MD, Thomas Sassard, MD, Abdel Shafy, MD, Mouhcine Madani, MD, and \\ Fadi Farhat, MD, PhD, Bron, France
}

Exposure of the mitral valve is a key success factor in minimally invasive mitral valve surgery (MIMVS) and a prerequisite for successful mitral valve repair. ${ }^{1}$ We report the concept of a new left atrial retractor (MitraXs, St Jude Medical Inc, Minneapolis, Minn) dedicated to MIMVS. The effectiveness of the device was evaluated in a prospective series.

\section{CLINICAL SUMMARY}

The MitraXs retractor is a pattern-cut polymer sheet that once deployed and secured by a pivoting rivet generates a conical volume (Figure 1). The device is a self-expanding, auto-adjusting, single-use left atrial retractor that does not require a supporting arm. The retractor has 2 sizes (A and B) according to valve diameter and 2 lengths (regular or " +") according to atrium depth to accommodate anatomic variations.

The introduction and deployment of the MitraXs device are key points. After the folding step, the MitraXs device is rolled and compressed from a conical shape to a cylinder with a reduced diameter creating a protruding triangular tab (Figure 1). By using a locking forceps, the MitraXs device is introduced via a minithoracotomy and an atriotomy, the tab with the rivet at the top loading the atrial septum first; then, with a clockwise rotation of a half turn, the device is progressively pushed into the left atrium in a screwing movement. When the MitraXs device is completely engaged in the left atrium, centered on the valve, with the rivet down in the posterior position, the forceps is released and the retractor deploys back into an optimal conical shape, working as an "expander" and maintaining the left atrium wide open in a symmetric manner.

The effectiveness of the MitraXs device was evaluated in 62 patients who consecutively underwent MIMVS during a 20-month period at the Louis Pradel Hospital. There

\footnotetext{
From the Louis Pradel Hospital, Cardiac Surgery and Transplantation, Bron, France. Disclosures: Olivier Jegaden reports fees and grant support from St. Jude.

Received for publication Oct 2, 2009; accepted for publication Nov 15, 2009; available ahead of print Feb 19, 2010.

Address for reprints: Olivier Jegaden, MD, Louis Pradel Hospital, Cardiac Surgery and Transplantation, 28 avenue du doyen Lepine, 69500 Bron, France (E-mail: olivier.jegaden@chu-lyon.fr).

J Thorac Cardiovasc Surg 2011;141:297-9

$0022-5223 / \$ 36.00$

Copyright (c) 2011 by The American Association for Thoracic Surgery doi:10.1016/j.jtcvs.2009.11.041
}
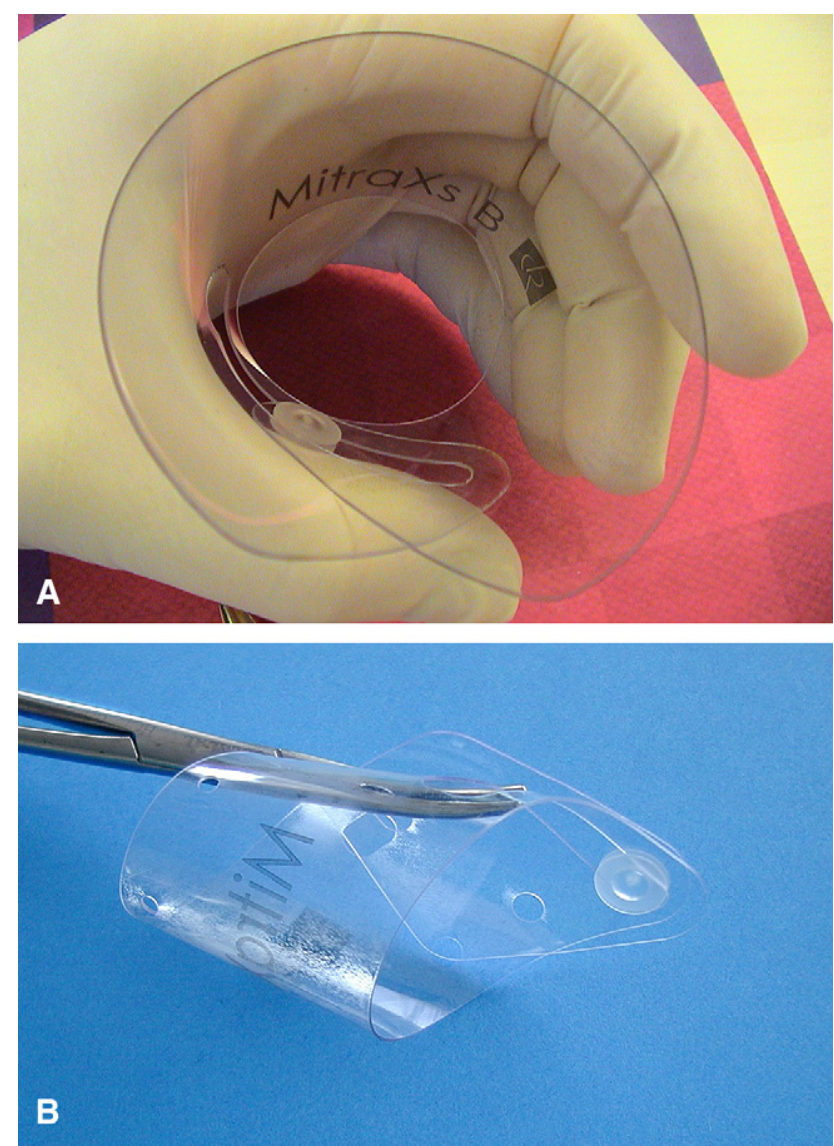

FIGURE 1. The MitraXs (St Jude Medical Inc, Minneapolis, Minn) is a self-supporting atrial retractor. A, In deployed position with 1 overmoduled pivoting rivet that makes its conical shape self-expanding and auto-adjusting. B, In rolled and compressed position, with triangular tab protruding out of the cylinder, which makes introduction into the left atrium easy.

were 21 female and 41 male patients with a mean age of $61 \pm 17$ years and a euroSCORE of $5.6 \pm 2.8$. The operation was a redo in 6 cases, with previous mitral valve surgery in 4 patients and previous coronary artery bypass grafting in 2 patients. The origin of mitral valve lesions was rheumatic in 11 patients, degenerative in 45 patients, endocarditis in 2 patients, and bioprosthesis dysfunction in 4 patients. The surgical procedure was based on the principles of MIMVS with a transthoracic aortic clamp. ${ }^{2}$ The MitraXs device was used in all cases as the primary indication for mitral valve exposure. The following were analyzed for each procedure: introduction of the retractor, size 

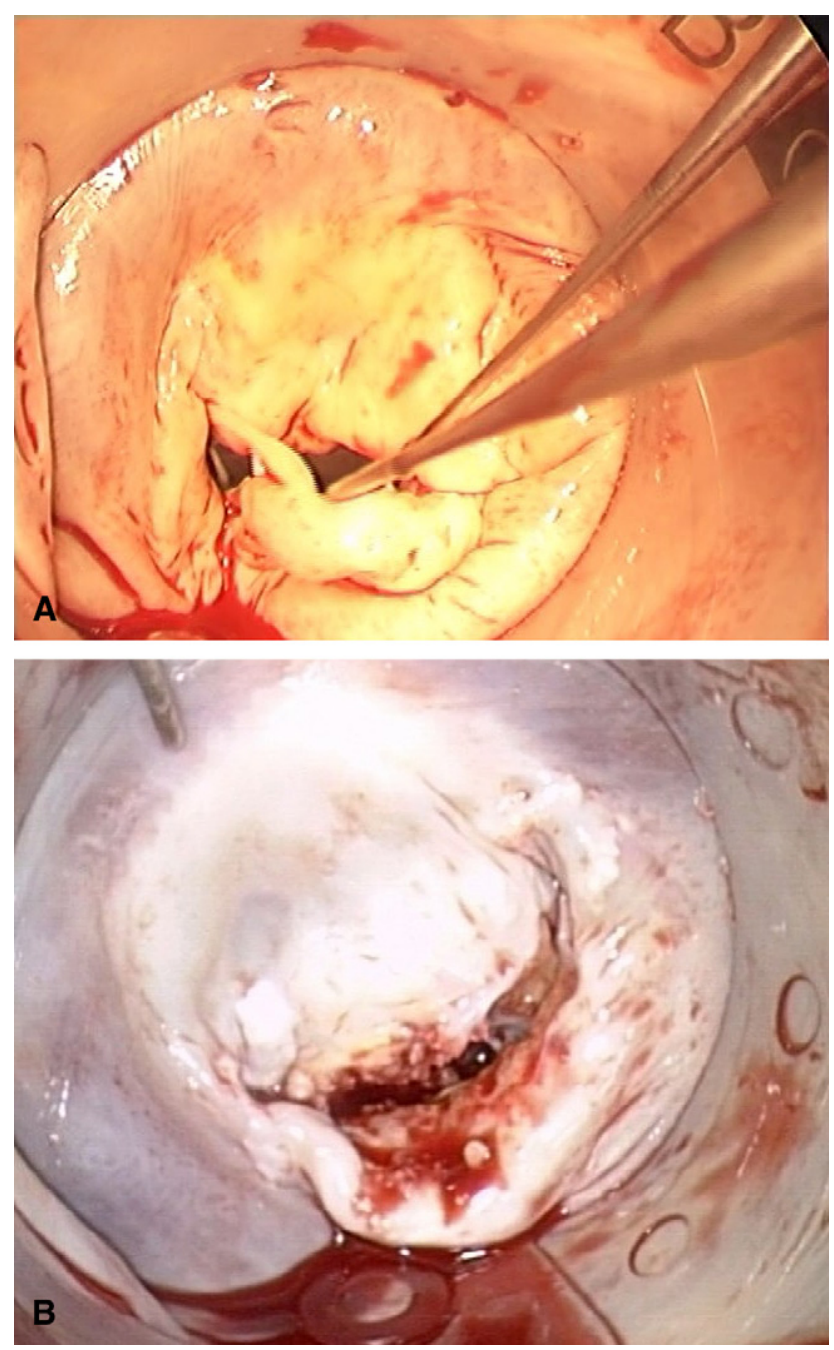

FIGURE 2. Intraoperative view. A, The mitral valve is nicely exposed without distortion, allowing easy and accurate analysis of the lesion. The left atrial muscle is homogeneously unfolded, providing space for suturing. $\mathrm{B}$, The mitral valve is visualized in its entirety with a large working space, making en bloc valve resection possible. Note the asanguineous operative field with the vent back. The MitraXs device may prevent tissue fragments from falling into the pulmonary veins.

suitability, conversion to blade retractor, exposure of the mitral valve, distortion of the anatomy, working space available around the valve, access for endoscopic instruments, need of repositioning or additional stitches to improve exposure, video and direct vision, and extraction of the device. At the end of the procedure, the surgeon proposed a global satisfaction index from 0 to 5 .

\section{RESULTS}

Mitral valve replacement was performed in 18 patients, and mitral valve repair was performed in 44 patients. An associated procedure was performed in 8 patients: myomectomy in 2 patients, atrial septal defect closure in 4 patients, and tricuspid valve repair in 2 patients. For mitral
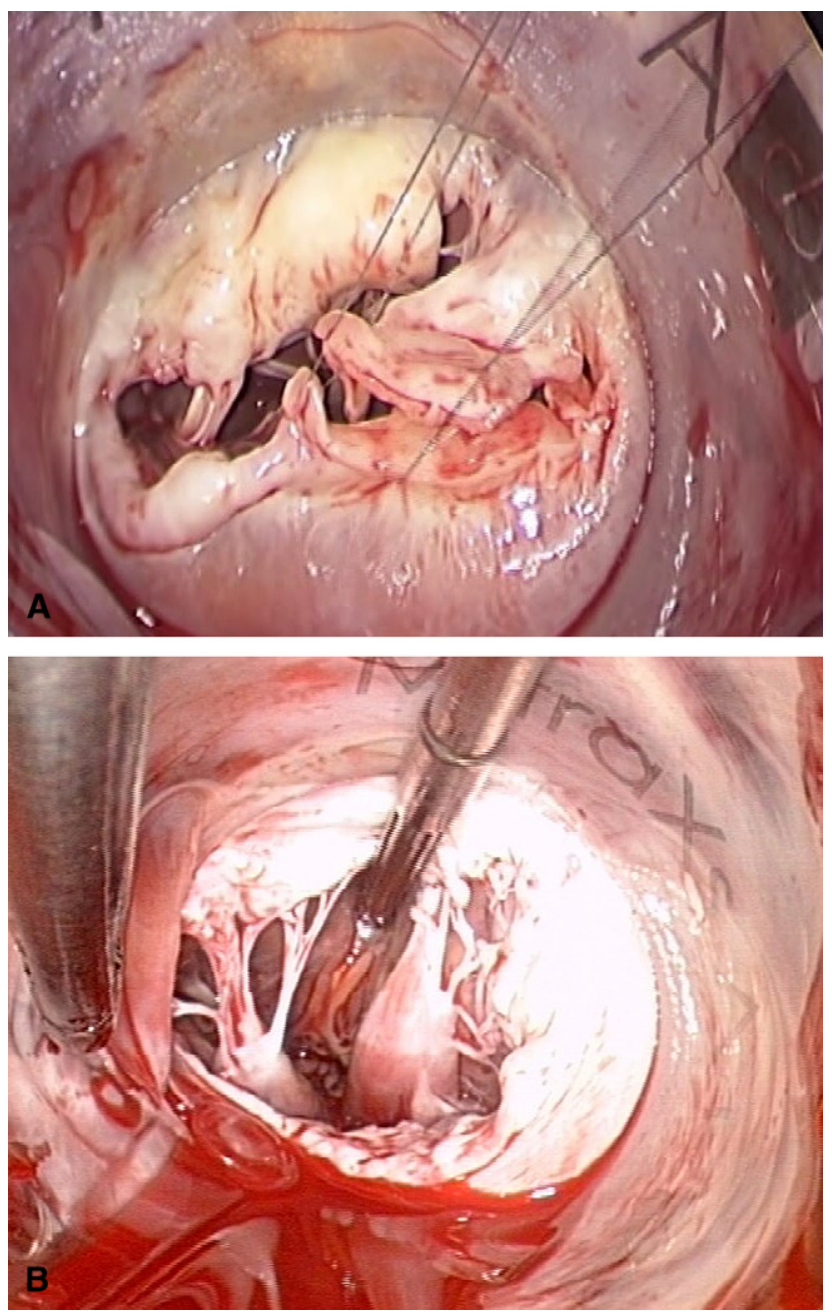

FIGURE 3. Intraoperative view. A, The MitraXs device is voluntarily centered on the posterior leaflet to make sliding plasty easier. Note the occluded area on anterior annulus that is accessible by sliding and repositioning. B, The MitraXs device is voluntarily pushed inside the mitral annulus to improve the exposure of papillary muscles for artificial chordae implantation.

valve repair, a posterior leaflet resection was performed in 28 patients and artificial chordae were used in 19 patients, mainly on the anterior leaflet; an annuloplasty using a complete semirigid ring was associated in all cases.

The introduction of the MitraXs device was fast and easy, with a mean placement time of $32 \pm 8$ seconds. There was a mismatch in 5 cases, and a smaller-sized MitraXs device was used. Size A was used in 34 patients (A, 21; A+, 13), and size $\mathrm{B}$ was used in 28 patients (B, 22; $\mathrm{B}+6)$. The MitraXs device was used in all procedures without an additional retractor or conversion to a different retractor. The exposure of the mitral valve, in regard to both leaflets and annulus, was estimated to be good to perfect in 47 cases, with the left atrial muscle homogeneously unfolded around the valve (Figure 2). In 15 cases of mitral valve repair, the 
anterior part of the annulus was partially hidden; in 9 cases, the repair of the well-exposed mitral valve was performed first and repositioning was done for ring implantation in the occluded area (Figure 3, A), and in 6 cases, sutures for ring implantation were placed first using sequential repositioning to downsize the valve diameter enough to be included in the internal diameter of the MitraXs device. Repositioning was easily performed with hooks in a sliding movement. In all cases, there was no distortion of the valve anatomy, which made the analysis of valve dysfunction easy and reliable in repair procedures. In 8 patients, to improve accessibility to the papillary muscles for artificial chordae implantation, the MitraXs device was first pushed inside the annulus to throw back the posterior leaflet (Figure 3, $B$ ). The same technique was used in 2 patients for associated myomectomy. In 4 patients, the MitraXs device (size+) slipped out of the left atrium during the procedure because of excessive traction on the annulus stitches, which progressively ejected the device; in 2 patients the device was successfully reintroduced into the left atrium, and in 2 patients the device was removed and ring implantation was finished without a retractor. An ideal straight line between the working port of the minithoracotomy and the plane of the mitral valve was obtained in most cases via a lateral thoracic approach; however, in 8 patients a "bayonet" effect was corrected using a traction stitch. In all patients, excellent direct vision was provided by the device. The vent placed in the pulmonary veins remained back without displacement or conflict. Repeat cardioplegia was performed with the MitraXs device in place, demonstrating no distortion of the aortic valve. In case of an associated right atrium approach for atrial septal defect or tricuspid surgery, the MitraXs device was used on the right side without the rivet engaged, working as a tent. Removal of the device was performed in a compressed cylindric shape $(n=51)$ or a flat shape after the rivet release $(n=$ 11). The global satisfaction index was $4.6 \pm 0.5$. All patients had a good outcome and were discharged from the hospital.

\section{DISCUSSION}

Optimal exposure of the mitral valve is a key point in MIMVS. Since our program was started in 1997, we have used the classic 2-piece blade retractor dedicated to such a procedure and observed its drawbacks related to the traction on the anterior leaflet with distortion of the mitral apparatus, the creation of atrial folds with large occluded areas, and the difficulty of not exposing the anterior annulus for ring implantation. ${ }^{3}$ The concept of the MitraXs device is based not on left atrial retraction but on left atrial expansion; thus, these drawbacks are not relevant. The association of a small left atrium with a dilated annulus was a difficult situation; the atrium diameter took the lead, and we had to accept a covered area in $24 \%$ of cases, no more than $10 \%$ on the anterior annulus. This drawback was offset by the freedom of the MitraXs device inside the left atrium, and repositioning was easy to modify the exposure according to the surgical stage. In the same manner, it was possible to focus the exposure on a specific area, such as the posterior annulus for sliding plasty or the papillary muscle for artificial chordae (Figure 3). In most cases, the MitraXs device provided optimal exposure and excellent direct vision. The global satisfaction index was high, demonstrating the effectiveness of the device; however, the MitraXs device has a few drawbacks. Size standardization may involve mismatch. Downsized or in case of excessive traction on annulus stitches, the MitraXs device may slip out of the left atrium.

\section{CONCLUSIONS}

The MitraXs is easy to use with excellent effectiveness. Since testing the device, we have adopted its use. According to our experience and the results of this series, the MitraXs makes the technique of MIMVS easier and more reproducible.

\section{References}

1. Taylor BS, Vanermen HK. Totally endoscopic mitral valve repair. Oper Tech Thorac Cardiovasc Surg. 2007;12:226-34.

2. Chitwood WR, Wixon CL, Elbeery JR, Moran JF, Chapman WHH, Lust RM Video-assisted minimally invasive mitral valve surgery. $J$ Thorac Cardiovasc Surg. 1997;114:773-82.

3. Ishikawa N, Sun YS, Nifong LW, Watanabe G, Chitwood WR. How to do it: importance of left atrial side retraction in robotic and minimally invasive mitral valve surgery. Heart Surg Forum. 2008;11:E270-1. 